\title{
Debris Disks in Nearby Young Moving Groups in the ALMA Era
}

\author{
Á. Kóspál and A. Moór \\ Konkoly Observatory, Research Centre for Astronomy and Earth Sciences, Hungarian \\ Academy of Sciences, PO Box 67, 1525 Budapest, Hungary \\ email: kospal@konkoly.hu, moor@konkoly.hu
}

\begin{abstract}
Many members of nearby young moving groups exhibit infrared excess attributed to circumstellar debris dust, formed via erosion of planetesimals. With their proximity and welldated ages, these groups are excellent laboratories for studying the early evolution of debris dust and of planetesimal belts. ALMA can spatially resolve the disk emission, revealing the location and extent of these belts, putting constraints on planetesimal evolution models, and allowing us to study planet-disk interactions. While the main trends of dust evolution in debris disks are well-known, there is almost no information on the evolution of gas. During the transition from protoplanetary to debris state, even the origin of gas is dubious. Here we review the exciting new results ALMA provided by observing young debris disks, and discuss possible future research directions.
\end{abstract}

Keywords. circumstellar matter, submillimeter: planetary systems, planet-disk interactions

\section{Introduction}

Nearly all young stars are encircled by massive circumstellar disks that serve as reservoirs for mass accretion and provide the necessary primordial gas and dust for the formation of planetesimals and planets. According to observations, gas-rich protoplanetary disks are depleted within about 10 million years and evolve into gas-poor, tenuous, dusty debris disks (e.g, Williams \& Cieza 2011). Debris disks are composed of second generation dust, where individual grains are rapidly removed mainly due to stellar radiation forces, but the dust is continuously replenished by collisional erosion or evaporation of previously formed planetesimals (Wyatt 2008).

Many young stars $(<200 \mathrm{Myr})$ in the solar neighbourhood belong to moving groups, i.e., gravitationally unbound, loose associations, whose members have common origin and move through space together (e.g., Kastner, this volume). A significant fraction of their members exhibits excess emission at infrared (IR) wavelengths (e.g., Zuckerman et al. 2011). While in some cases this may come from long-lived protoplanetary disks, the majority can be attributed to circumstellar debris dust. Many well-studied debris systems, e.g., $\beta$ Pic, AU Mic, HR 4796A, belong to young moving groups. With their proximity and well-dated ages between 8 and $200 \mathrm{Myr}$, these groups offer an excellent laboratory for studying the evolution between the primordial and debris stages, as well as the early evolution of debris dust and, thereby, of planetesimal belts. This time range overlaps with the expected last phase of the formation of terrestrial planets (Raymond et al. 2014) and the initiation of collisional cascade and production of observable debris material in the outer disk regions (Kenyon \& Bromley 2008). However, most debris disks around members of nearby young moving groups have remained spatially unresolved so far, providing only limited information about disk properties. 
With its unprecedented sensitivity and spatial resolution, the recently commissioned Atacama Large Millimeter/submillimeter Array (ALMA) is opening a new era in the investigation of debris disks by allowing to observe both the thermal emission of cold dust and the rotational lines of different gas molecules (if present). By studying ALMA continuum data we can map the spatial distribution of mm-sized grains, which dominate the disk emission at submm/mm wavelengths. These large dust grains are less subject to dynamical removal effects than smaller grains and are therefore the best tracers of the structure of the parent planetesimal belts. By determining the location and extent of these belts, we can put contraints on planetesimal formation and evolution models and the underlying dynamical processes. It also allows to probe traces of the interaction between giant planets and the disk. Molecular line observations with ALMA allows to measure the amount of gas in debris disks and how it evolves during the transition from protoplanetary to debris state, a largely unexplored field so far. In the following we review open questions concerning the dust and gas content of young debris disks, the latest ALMA results in the field, and promising future directions.

\section{Stirring of debris disks}

Planetesimals in protoplanetary disks are on low eccentricity and low inclination orbits because of the damping effect of gas. Therefore, collisions between them occur at low relative velocities resulting in the merging of bodies, even after the amount of gas decreases. For destructive collisions with effective dust production, the motion of planetesimals needs to be stirred. In the self-stirring scenario proposed by Kenyon \& Bromley (2008), Pluto-sized bodies embedded in the outer disk can initiate a collisional cascade by perturbing the orbits of neighbouring smaller planetesimals. As large planetesimals build up slower at larger disk radii, the collisional cascade is ignited in the inner disk first and then the active dust production propagates outward. As the disk evolves, planetesimals in the inner regions are ground down, leading to a decline in the local dust production. This results in an outwardly increasing dust surface density whose maximum coincides with the region where Pluto-sized bodies have just been formed (Kennedy \& Wyatt 2010). This is very different from the surface density profiles observed in protoplanetary disks. The pace of the outward propagation of the stirring front depends on the disk surface density: in an initially denser, more massive disk, the outwards spread is faster (Kenyon \& Bromley, 2008). A giant planet or a stellar companion can also excite the motion of planetesimals via its secular perturbations (planetary stirring, Wyatt 2005). If the perturber is located closer to the star than the planetesimal belt, then this mechanism also results in an inside-out disk stirring whose timescale could be even shorter than that of self-stirring (Mustill \& Wyatt 2009).

Observational verification of specific aspects of this picture, however, are not yet conclusive. By mapping the spatial distribution of mm-sized debris dust grains, we can evaluate several predictions of stirring models. Using ALMA, MacGregor et al. (2013) and Ricci et al. (2015) successfully resolved the radial density surface density profiles in two young broad debris disks around AU Mic and HD 107146. In both cases a rising profile was found at least in the outer regions of the disks, consistently with the predictions of stirring models. The site of active dust production in stirring models is expected to propagate outward as a function of age. Previous observations have already suggested a weak correlation between disk radii and ages (e.g., Rhee et al. 2007; Eiroa et al. 2013). However, disk radii in these studies were estimated from their characteristic temperature and the stellar luminosity, assuming blackbody grains. Because of the well-known degeneracy between the equilibrium temperature and size of grains, these estimates are not 
unambigous (e.g. Krivov 2010). By measuring debris disk sizes with ALMA in well-dated systems with different ages, e.g., in different young moving groups, this predicted trend can be reliably verified. In unusually extended young debris disks, the self-stirring model would require too massive initial disks. In such cases, the self-stirring scenario is unfeasible, thus, they can be considered as prime candidates for planetary stirring. Indeed, the debris disks around HR 8799, $\beta$ Pic, and HD 95086 - systems with known outer giant planets - fall into this category (Moór et al. 2015). This principle can be used to search for additional planetary stirring candidates with ALMA. Since the potential targets are young, using advanced adaptive optics systems such as the Gemini Planet Imager or VLT/SPHERE (Macintosh et al. 2008, Beuzit et al. 2010), even the perturber(s) could be identified.

\section{Signatures of planet-disk interaction}

Debris dust grains and the planetesimals from which they are derived are the smallest constituents of a planetary system that may include more massive planets as well. As the system evolves, the planet(s) and the planetesimal disk can interact in many ways. The different types of gravitational perturbations can cause various kind of footprints, such as offsets, spirals, gaps, or clumps in the disk. Secular perturbations from a misaligned or eccentric planet can cause warps or tightly wound spirals in the disk (Matthews et al. 2014a). These structures then propagate through the disk, as the gravitational effect of the planet extends to outer and outer regions. By forcing the distant planetesimals on intersecting orbits, this may result in more frequent collisions that occur with higher relative velocities, eventually leading to the initiation of a collisional cascade. After a time, the secular perturbations from an eccentric planet make the whole disk also to be eccentric (e.g.,Kalas et al. 2005).

Resonant perturbations occur when dynamical frequencies, typically of the mean motions, are a simple integer ratio of each other. Resonances can lead to either stabilization or destabilization of the orbits, causing overpopulated or underpopulated regions in a debris disk (Matthews et al. 2014a, and references therein). As a consequence of overlapping first-order mean-motion resonances, the region close to the planet's orbit becomes chaotic, and dust particles orbiting in this zone are short-lived. Therefore, the chaotic zone is evacuated and a gap is formed. In our Solar System, the Kirkwood gaps in the asteroid belt are formed via such mean motion resonances with Jupiter. Overpopulated regions can also be formed via resonant captures, such as the Trojan asteroids (in 1:1 resonance with Jupiter) or the Plutinos (in 3:2 resonance with Neptune) in our Solar System. In a debris disk, two different migration mechanisms are proposed to lead to resonant clumps in the dust distribution. Outward migration of a planet can trap planetesimals into its external resonances, the erosion of these planetesimals then produce an enhanced dust population. Smaller grains can escape from these regions because of radiation pressure. Large particles, however, are trapped in the resonance and cause a local enhancement that can be traced in submillimeter/mm maps of the disk (Wyatt, 2003). Alternatively, in those tenuous debris disks where the timescale of Poynting-Robertson drag is shorter than the collisional timescale, dust grains produced in an outer planetesimal belt can spiral inward due to drag forces into the mean-motion resonances with an inner planet (Kuchner \& Holman, 2003).

ALMA observations of Fomalhaut's disk showed that the mm-sized dust is concentrated in a very narrow, vertically thin ring, whose inner and outer edges are sharply truncated (Boley et al. 2012). This ring morphology can be best explained with the presence of two shepherding planets located at the inner and the outer edges, which are likely to be less 
massive than $3 \mathrm{M}_{\oplus}$. HD 107146 is encircled by a very broad debris disk. ALMA images of the radial distribution of dust revealed a decrease in the surface brightness profile at intermediate radii (Ricci et al. 2015). One possible interpretation is that this is related to a gap carved by a few Earth-mass planet in the dusty disk.

Previously, based on spatially resolved millimeter images of nearby bright debris disks around Vega, $\epsilon$ Eri, and HD 107146, several clumpy structures were reported and the presence of these inhomogeneities were linked to large grains trapped in mean motion resonances with unseen planets (Holland et al. 1998; Greaves et al. 1998; Corder et al. 2009). Most of these structures, however, turned out to be dubious, because subsequent more sensitive and higher resolution observations could not confirm their presence (e.g., Piétu et al. 2011; Hughes et al. 2011). Thanks to its excellent sensitivity, ALMA can detect possible resonant structures of even lower contrast in the brightness distribution of debris disks. Recent ALMA maps of $\beta$ Pic revealed a bright region in the southwestern part of the disk. This clump be seen both in the distribution of CO gas and mm-sized dust grains (Dent et al. 2014) and it also coincides well with an enhancement of micron-sized grains observed at resolved mid-IR images (Telesco et al. 2005). Both the gas and dust in $\beta$ Pic may have secondary origin. Based on current data, the clump can be attributed to frequently colliding icy planetesimals trapped in a mean motion resonance with an outwardly migrating planet. Alternatively, the clump may be a result of a recent collision between two Mars-sized icy comets (Dent et al. 2014).

The observed disk asymmetries and structures can be used to deduce the orbit and other parameters of the planet(s) responsible for them and can also provide an opportunity to constrain the dynamical history of the planetary system. In young systems, wide separation giant planets predicted in this way can be confirmed using new generation adaptive optics systems. Moreover, such observations of the perturbers will be able to help in the evaluation and improvement of methods we use in the interpretation of structures seen in the dust distribution. Indeed now we may already know two potential benchmark systems. HR 8799 and HD 95086 are two young A-type stars with outer giant planets detected by direct imaging (Marois et al. 2010; Rameau et al. 2013). Both systems harbor a warm inner dust belt and a broad cold outer disk. The giant planet(s) are located between the two dusty regions (Matthews et al. 2014b; Moór et al. 2013a; Su et al. 2009, 2015). ALMA observations of these disks are ongoing.

\section{Gas in young debris disks}

Debris disks are expected to be gas-poor systems with a gas-to-dust ratio significantly lower than in protoplanetary disks. The detection of the presumably little amounts of gas is a challenging task. Despite substantial efforts, we know only eight debris disks where gas has been detected. In the pre-ALMA era, the edge-on orientation of disks around $\beta$ Pic and HD 32297 allowed the detection of their gas content via absorption lines (Slettebak et al. 1975; Hobbs et al. 1985; Redfield 2007). The gaseous disks around HD 172555, HD 181296, and AU Mic were identified based on O I , C II, and fluorescent $\mathrm{H}_{2}$ line emissions, respectively (Riviere-Marichalar et al. 2012, 2014; France et al. 2007), while CO gas has been observed in disks around 49 Ceti, HD 21997, and HD 131835 (Zuckerman et al. 1995; Moór et al. 2011; Moór et al. in prep). All of these systems are probably younger than $50 \mathrm{Myr}$, and with the exception of HD 32297 they belong to young moving groups or associations. Interestingly, apart from the M-type AU Mic, all of the host stars are A-type. The origin of gas in these disks is uncertain. In most cases the gas might be secondary, i.e., similarly to dust grains it is continuously replenished via erosion of larger bodies. Collisions between icy planetesimals, evaporation of comets 
or icy grains, and photon-induced desorption of solids can lead to gas release. However, because of their youth, we cannot exclude that some of these disks are hybrid in the sense that they retain their residual primordial gas, while the dust component have secondary origin.

The first observations of gaseous debris disks with ALMA provided examples of both types. By mapping the dust and gas in the disk around $\beta$ Pic, Dent et al. (2014) found that the spatial distribution of the two components is similar, both showing a characteristic bright clump in the southwest part of the disk. The short lifetime of CO and other dynamical arguments make it probable that the gas in this system has secondary origin, probably indicating intense collisional activity (Fernández et al. 2006; Dent et al. 2014). ALMA observations of HD 21997 showed that the CO gas is not co-located with the mm-sized dust and thereby the planetesimals, but there is a dust-depleted inner gas disk (Kóspál et al. 2013; Moór et al. 2013b). Moreover, the disk's total CO content is more than a thousand times larger than that of $\beta$ Pic. These cannot be explained with secondary gas models, implying that this disk instead harbors residual primordial gas and is the first example of a hybrid disk (Kóspál et al. 2013). HD 21997 may be a unique object or may be the tip of an iceberg of many fainter hybrid disks. In the latter case, the gas-rich phase of disk evolution could be significantly longer than previously thought, also affecting the formation of planets.

\section{Conclusions}

Before ALMA, less than a dozen debris disks were spatially well resolved at millimeter wavelengths. These studies constituted the first steps towards understanding the evolution of young debris systems. ALMA is now in the process of multiplying the sample of resolved debris disks, thereby allowing to study more general trends. The first observations have already produced remarkable results. ALMA confirmed that the rising brightness profile seen in certain debris disks is consistent with the stirring models. It also identified disks where the derived initial mass was too high for the self-stirring scenario, pointing to planetary stirring. It imaged structures that hint at so-far unknown planets shepherding the dust or clearing gaps. Finally, it detected a large amount of molecular gas, leading to the discovery of the first hybrid disk where the dust is secondary but the gas component is still primordial. In the coming years, using an increasingly larger sample, we will be able to answer several fundamental questions concerning the dust and gas content of debris disks. Dust continuum observations will be used to, e.g., verify whether the location of the dust-producing region expands with time, to identify asymmetric dust distributions and their relationship with planets. Gas line observations with ALMA are the best way to study the cold gas content of debris disks, providing a possibility to study how the gas evolves, and what mechanism produces the gaseous material. These exciting new results ahead of us will transform the research of debris disks, and - in connection with the age and environmental information provided by their membership in young moving groups - will shed light onto a period of very active planet formation.

\section{Acknowledgment}

This work was supported by the Momentum grant of the MTA CSFK Lendület Disk Research Group. A.M. acknowledges support from the Bolyai Research Fellowship of the Hungarian Academy of Sciences. 


\section{References}

Beuzit, J.-L., Boccaletti, A., Feldt, M., et al. 2010, Proceedings of the workshop "Pathways Towards Habitable Planets", V Coudé du Foresto, D. M. Gelino, and I. Ribas, eds., p. 231

Boley, A. C., Payne, M. J., Corder, S., et al. 2012, ApJL, 750, L21

Corder, S., Carpenter, J. M., Sargent, A. I., et al. 2009, ApJL, 690, L65

Dent, W. R. F., Wyatt, M. C., Roberge, A., et al. 2014, Science, 343, 1490

Eiroa C., et al. 2013, A\&̈A, 555, A11

Fernández, R., Brandeker, A., \& Wu, Y. 2006, ApJ, 643, 509

France, K., Roberge, A., Lupu, R. E., Redfield, S., \& Feldman, P. D. 2007, ApJ, 668, 1174

Greaves, J. S., Holland, W. S., Moriarty-Schieven, G., et al. 1998, ApJL, 506, L133

Hobbs, L. M., Vidal-Madjar, A., Ferlet, R., Albert, C. E., \& Gry, C. 1985, ApJ, 293, L29

Holland, W. S., Greaves, J. S., Zuckerman, B., et al. 1998, Nature , 392, 788

Hughes, A. M., Wilner, D. J., Andrews, S. M., et al. 2011, ApJ, 740, 38

Kalas, P., Graham, J. R., \& Clampin, M. 2005, Nature, 435, 1067

Kenyon, S. J. \& Bromley, B. C. 2008, ApJS, 179, 451

Kennedy G. M. \& Wyatt M. C. 2010, MNRAS, 405, 1253

Kóspál, Á., Moór, A., Juhász, A., et al. 2013, ApJ, 776, 77

Krivov A. V. 2010, RAA, 10, 383

Kuchner M. J. \& Holman M. J. 2003, ApJ, 588, 1110

MacGregor, M. A., Wilner, D. J., Rosenfeld, K. A., et al. 2013, ApJL, 762, L21

Macintosh, B. A., Graham, J. R., Palmer, D. W., et al. 2008, Proceedings of the SPIE, N. Hubin, C. E. Max, P. L Wizinowich, eds., Vol 7015, article id. 701518

Marois C., Zuckerman B., Konopacky Q. M., Macintosh B., \& Barman T. 2010, Nature, 468, 1080

Matthews B. C., Krivov A. V., Wyatt M. C., Bryden G., \& Eiroa C. 2014a, Protostars and Planets VI, H. Beuther, R. Klessen, C. Dullemond, Th. Henning, eds., p. 521-544.

Matthews B., Kennedy G., Sibthorpe B., Booth M., Wyatt M., Broekhoven-Fiene H., Macintosh B., \& Marois C. 2014b, ApJ, 780, 97

Moór, A., Ábrahám, P., Juhász, A., et al. 2011, ApJ, 740, L7

Moór, A., et al. 2013a, ApJ, 775, L51

Moór, A., Juhász, A., Kóspál, Á., et al. 2013b, ApJ, 777, 25

Moór, A., Kóspál, Á., Ábrahám, P., et al. 2015, MNRAS, 447, 577

Mustill A. J. \& Wyatt M. C. 2009, MNRAS, 399, 1403

Piétu, V., di Folco, E., Guilloteau, S., Gueth, F., \& Cox, P. 2011, A\&A, 531, L2

Rameau J., et al. 2013, ApJ, 779, L26

Raymond S. N., Kokubo E., Morbidelli A., Morishima R., \& Walsh K. J. 2014, Protostars and Planets VI, H. Beuther, R. Klessen, C. Dullemond, Th. Henning, eds., p. 595-619.

Redfield, S. 2007, ApJ, 656, L97

Rhee J. H., Song I., Zuckerman B., \& McElwain M. 2007, ApJ, 660, 1556

Ricci, L., Carpenter, J. M., Fu, B., et al. 2015, Apj, 798, 124

Riviere-Marichalar, P., Barrado, D., Augereau, J.-C., et al. 2012, A\&A, 546, L8

Riviere-Marichalar, P., Barrado, D., Montesinos, B., et al. 2014, A\&A, 565, A68

Slettebak, A. 1975, ApJ, 197, 137

Su, K. Y. L., Rieke, G. H., Stapelfeldt, K. R., et al. 2009, ApJ, 705, 314

Su, K. Y. L., Morrison, S., Malhotra, R., et al. 2015, ApJ, 799, 146

Telesco, C. M., Fisher, R. S., Wyatt, M. C., et al. 2005, Nature, 433, 133

Williams, J. P. \& Cieza, L. A. 2011, ARA\&A, 49, 67

Wyatt, M. C. 2003, ApJ, 598, 1321

Wyatt, M. C. 2005, A\&A A, 440, 937

Wyatt, M. C. 2008, ARA $G A, 46,339$

Zuckerman, B., Forveille, T., \& Kastner, J. H. 1995, Nature, 373, 494

Zuckerman B., Rhee J. H., Song I., \& Bessell M. S. 2011, ApJ, 732, 61 